\title{
THE BASIC KARYOTYPE OF RYE (SECALE CEREALE) ANALYSED WITH GIEMSA AND FLUORESCENCE METHODS
}

\author{
CANIO G. VOSA \\ Botany School, Oxford
}

Received 26.iii.74

\begin{abstract}
SUMMARY
The basic karyotype of Rye (Secale cereale L.) has been analysed with several fluorochromes and with a Giemsa staining technique. Only the fluorochrome Hoechst 33258 and the Giemsa technique were successful in differentiating the heterochromatic segments.

Five cultivated varieties were studied. In the haploid set there are 11 large and two small terminally located bands, four small intercalary bands and a variable band adjacent to the nucleolar constriction on chromosome VII which are constant for all varieties. Five more very small intercalary bands occur sporadically in all the varieties. All the chromosomes possess thin centromeric bands.
\end{abstract}

\section{INTRODUCTION}

RYE is the hardiest of the cereals and its cultivation reaches beyond the Arctic Circle. It is of considerable importance in Russia, Sweden, Poland, the Netherlands and Belgium. In Britain, Rye has ceased to be of any great importance since the change to wheaten bread in the eighteenth century. Compared with other cereals, Rye has fewer varieties and these are not very uniform because of cross-pollination which is the rule in Rye but the exception in other cereal crops.

It probably occurred as a weed in fields before being accepted as a crop rather later in the history of civilisation. Its oldest archaeological records date from the first Iron Age (Halstatt period, c. 900-700 B.c.).

The first chromosome counts are those of Nemec (1910) as cited by Emme (1928), giving 18 chromosomes for endosperm cells. Other reports (Westigate, see Stolze, 1925) and Spillman (cf. Hector, 1934) gave $2 n=12$ and Nakao (1911) found $n=8$ in the P.M.C.s. The first correct chromosome count is that of Sakamura (1918), but because of the discrepancy between the previous counts, owing perhaps to the presence of supernumerary chromosomes, the true haploid number remained uncertain until the works of Nikolaieva (1924) and Stolze (1925). Lewitsky (1931) was the first to study the morphology of Rye chromosomes from somatic tissues (see Jain, 1960 for bibliography). Other karyological studies include those of Pathak (1940), Levan (1942) and Sybenga and Wolters (1972), while Lima de Faria $(1952 a, b)$ made a very careful analysis of the pachytene chromosomes. Lima de Faria (1959) and Darlington and Haque (1966) have studied the sequence of DNA synthesis in Rye chromosomes. Recently Sarma and Natarajan (1973), Merker (1973) and Verma and Rees (1974) have demonstrated the differential staining of the heterochromatic regions 
of Rye chromosomes with various methods. The present study is concerned with an analysis of the basic karyotype of Rye with a modified denaturation-reannealing technique using Giemsa as a stain and with fluorochroming with Quinacrine (Q), Quinacrine Mustard (QM), Ethidium Bromide (EB) and with the bibenzimidazole Hoechst 33258.

\section{Materials AND Methods}

The material consisted of seeds of five diploid cultivated varieties of Rye of which two were from Afghanistan and the others from Europe. The European varieties included two winter long-straw types: Norma and Lovászpatonai and a winter short-straw type: Cortina. Cortina and Norma originated from the seed firm of F. v. Lochow-Petkus of Western Germany and Lovászapatonai originated from Hungary. The seeds were germinated on filter paper in plastic petri dishes or in small pots in fine peat. The germination time is about 36 hours at $20^{\circ} \mathrm{C}$.

The cytological methods are basically those of Vosa and Marchi (1971, 1972).

Actively growing root-tips were pretreated in 0.05 per cent colchicine at room temperature for 3-4 hours and then fixed overnight or at least for 5 hours in 1:3 acetic-alcohol. For the Giemsa stained preparations, the fixed roots were hydrolysed for 25-30 seconds in $1 \mathrm{~N} \mathrm{HCl}$ at $60^{\circ} \mathrm{C}$. and then washed and kept in distilled water. Squashing was performed in 45 per cent acetic acid on a clean slide and the coverslip was coated with a thin layer of glycerin-albumen, dried over a spirit flame and placed face downward on the squashed material. After pressing firmly between layers of filter paper, the preparations were inverted in a ridged dish of absolute alcohol. The coverslips will separate from the slides after a while and after rinsing in alcohol and air-drying, they were immersed in a saturated solution of Barium hydroxide for 5 minutes at room temperature. The temperature of the Barium treatment is very important and for the best results should never fall below $18^{\circ} \mathrm{C}$. Optimum temperature is between $18^{\circ}$ and $22^{\circ} \mathrm{C}$. Higher temperatures may cause excessive swelling and distortion of the chromosomes. After this treatment the coverslips were thoroughly washed in distilled water in order to eliminate all traces of alkali and while still wet they were immersed in $2 \mathrm{X} \mathrm{SSG}$ at $60^{\circ} \mathrm{C}$. for 45-60 minutes. Then, after rinsing in distilled water, they were placed in dishes of 0.5 per cent Giemsa (Gurr's) at $p \mathrm{H} 6.8$ for 20-30 minutes. It is useful to monitor the staining for optimum results as different batches of stain vary in their reaction and overstaining is to be avoided. Mounting was in DPX or in neutral balsam. For the fluorescence preparations, the root tips were squashed directly in 45 per cent acetic acid without previous hydrolysis. The subsequent procedure was exactly as for the Giemsa preparations as far as the separation of the coverslips from the slides. After separation, the coverslips were rinsed in alcohol and without air drying placed in an alcoholic solution of the fluorochromes. The concentration of the fluorochromes in absolute alcohol was 0.5 per cent for $Q, 0.01$ per cent for QM, 0.02 per cent for EB and 0.02 per cent for Hoechst 33258. Staining was carried out in all cases at room temperature for 3-5 minutes. After rinsing in absolute alcohol and air drying, the coverslips were mounted in water or in $1: 1$ water-glycerol and observed immediately with the fluorescence 

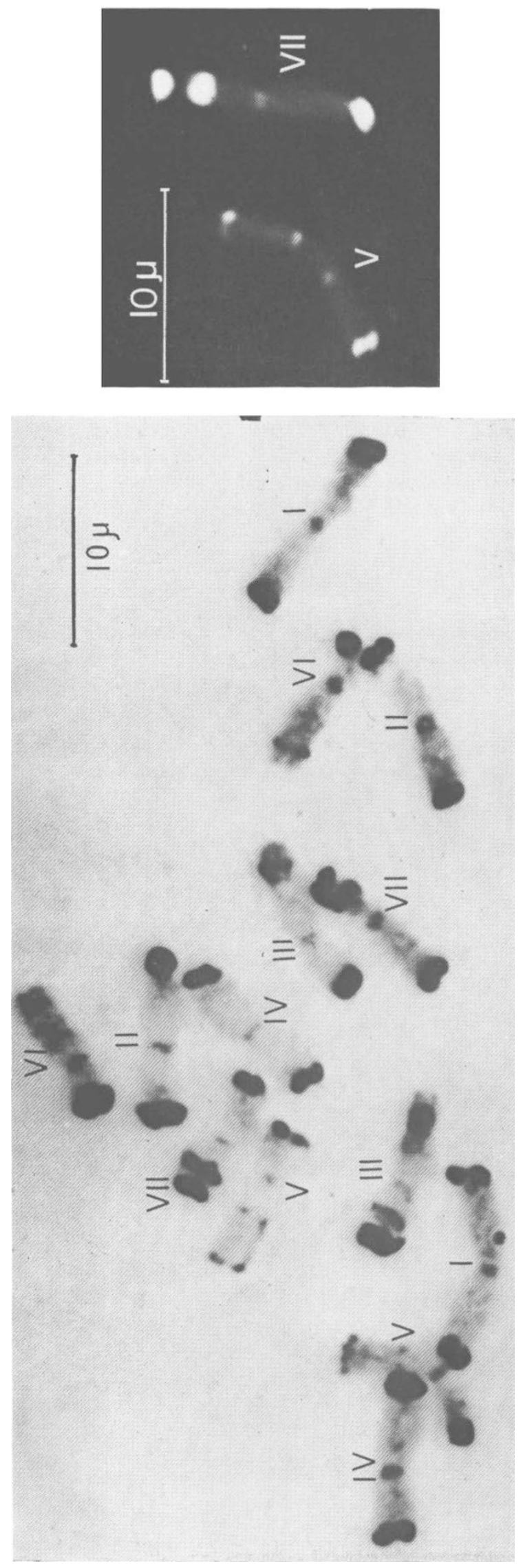

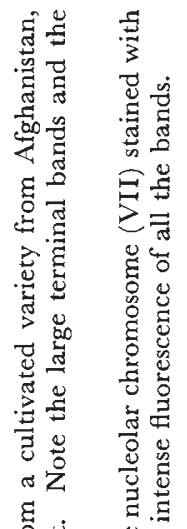

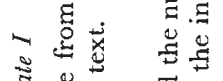

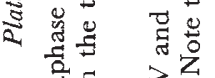
要 $>$ छ․ छ

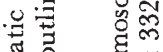
킁 दू.

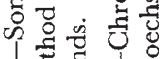

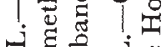
ㄴ. 욜 षै. 渮过 క్․․ त. 它它 
microscope. The fluorescence of the preparations stained with Hoechst 33258 is more stable than that of $Q$ or QM and it does not fade appreciably even after prolonged irradiation.

All the observations were made with a Zeiss Ultraphot microscope. For fluorescence, barrier filters $53 / 44$ or 50 and exciter filter BG 12 were used. Kodak Microfile and TRI-X Pan films were used for ordinary light and fluorescence micrographs respectively.

\section{Results}

Staining with $Q, Q M$ and EB did not result in successful banding and gave uniform fluorescence along the chromosomes. Only the Giemsa

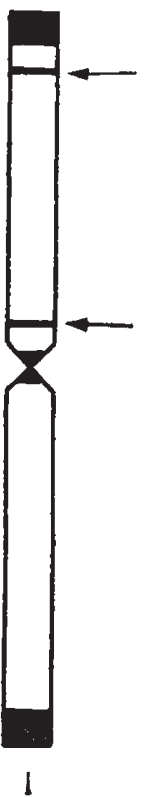

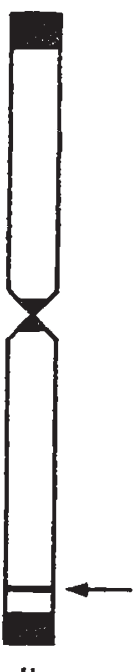

II

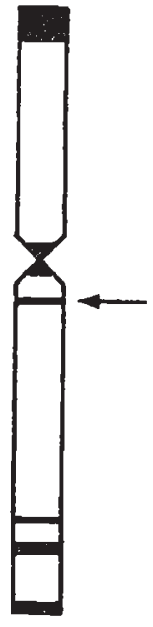

III

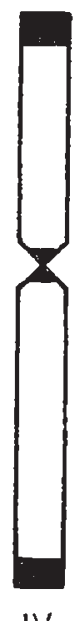

IV

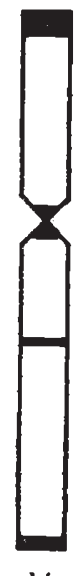

$\mathrm{V}$

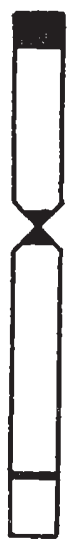

VI
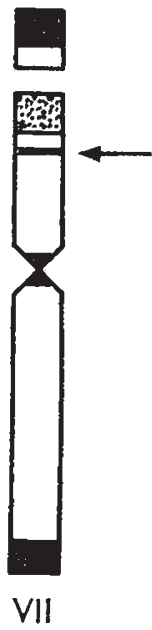

Fig. 1.- Secale cereale L.-The ideogrammatic representation of the haploid complement. The ideogram includes the pooled informations obtained in the study of 30 plants from five cultivated varieties. The five small occasional bands are arrowed and the variable band in chromosome VII is stippled. The chromosomes have been arranged in order of decreasing length.

banding technique (plate I, fig. a) and the fluorochrome Hoechst 33258 (plate I, fig. b) gave satisfactory and highly repeatable banding patterns. The results are very similar to those obtained by Sarma and Natarajan (1973), Merker (1973) and Verma and Rees (1974) in the same species. However, their findings differ in details from those presented here: Sarma and Natarajan were able to distinguish only five chromosome types and, for some reason, failed to detect the thin but very conspicuous centromeric bands.

The basic haploid karyotype of Rye is illustrated in fig. 1. The ideogram contains the pooled informations obtained in the analysis of a total of 30 plants from the five varieties. A slight variation in chromosome size 
and in arm ratio as well as in the size of the bands was observed but no specific varietal differences were found.

In the haploid set 11 large and two small terminal bands and four small intercalary bands are constant in all varieties studied. In addition, five more small intercalary bands are occasionally found in some plants. All varieties possess a band adjacent to the nucleolar constriction in the short arm of chromosomes VII. This band is very variable in size and has a lower intensity of stain than the other bands. All the chromosomes have thin centromeric bands which do not seem to show any consistent variation in size.

\section{Chromosome types}

I. Two thick bands located terminally in each arm. In the short arm two very thin bands, one very near the terminal band and the other proximal to the centromere, are sometimes present. These thin bands occur sporadically in all the varieties and may be present together or, more often, singly.

II. Two thick bands located terminally one in each arm. In addition, one thin band near the terminal band in the long arm is occasionally found in some varieties.

III. One thick band terminally in the short arm, two thin bands (one slightly larger than the other) in the distal third and one thin band terminally in the long arm.

IV. One thick band located terminally in each arm.

$\mathrm{V}$. One thick band terminally in the short arm and two thin bands in the long arm, one in a median position and the other terminal.

VI. One thick band terminally in the short arm and one thin band located in the distal third of the long arm.

VII. The nucleolar chromosome has three bands: one terminal in the long arm, one on the satellite covering about two-thirds of its distal end and one very variable in size and with a lower intensity of stain than the other bands, adjacent to the nucleolar constriction in the short arm. In most plants in all varieties a very thin band is found near the variable band in the short arm.

The location of the heterochromatic segments agree very well with the chromomere distribution pattern of the pachytene chromosomes. This includes the "gradient" either side of the centromere, as described by Lima de Faria $(1952 a, b)$, but the size of the centric segments observed with Giemsa staining and with fluorochroming appears to be much smaller than implied by Lima de Faria studies. The thick terminal bands correspond closely with the late replicating regions detected with autoradiographic techniques by Lima de Faria (1959), Darlington and Haque (1966), and Ayonoadu and Rees (1973). Lima de Faria (loc. cit.) considered the centromeric heterochromatin as regions of late DNA replication. However, Ayonoadu and Rees (loc. cit.) attributed the heavy labelling around the centromere in metaphase chromosomes, not to a late completion of DNA synthesis but to a higher degree of condensation of these regions at this stage.

An analysis of prophasic stages indicates that whilst the terminal segments remain condensed throughout the mitotic cycle, most of the intercalary bands and the centromeric bands are less condensed appearing to be at an intermediate level between the terminal heterochromatin and the euchromatin. 
This suggests that the centromeric heterochromatin and some of the intercalary bands are different from the terminal bands. Taking into account the lower stainability of the band adjacent to the nucleolar constriction in the short arm of chromosome VII and including euchromatin, there appear to be four chromatin classes in the chromosome complement of Rye.

Parallel studies in several diploid and tetraploid wheat species (Triticum spp.) as well as tetraploid and hexaploid cultivated varieties have indicated the presence of thin terminal, intercalary and centric bands in most chromosomes.

As Sarma and Natarajan (1973) and Merker (1973) have noted in their investigations in Triticale, it is possible to distinguish between the chromosomes of the parent species since only Rye possesses thick terminal bands.

A preliminary study in Barley (Hordeum vulgare) chromosomes has revealed that in this species the distribution of heterochromatin is almost exclusively proximal, with comparatively thick centromeric bands and thin intercalary bands.

The karyotype of Rye as presented in this study should prove very useful as a basis for further chromosome studies including gene mapping and, by providing chromosome markers, recombination studies and breeding experiments.

Acknowledgments.-I wish to thank Dr K. R. Lewis for his interest and for helpful discussions during the course of this work and $\mathrm{Dr}$ H. Loewe of Farbwerke Hoechst, Frankfurt, Main, for the kind gift of the bibenzimidazole " 33258 Hoechst".

\section{REFERENCES}

AYONOADU, U., AND REEs, H. 1973. DNA synthesis in Rye chromosomes. Heredity, 30, 233-240.

DARLINGTON, C. D., AND HAQUE, A. 1966. Organization of DNA synthesis in Rye chromosomes. Chromosome Today, 1, 102-107.

емме, н. 1928. Karyologie der Gattung Secale L. Zschr. ind. Abst. Vererb. L., 47, 99-124. HECTOR, J. 1934. Introduction to the Botany of Field Crops. Vol. 1 Cereals. Central News Agency Ltd. Johannesburg.

JaIN, s. K. 1960. Cytogenetics of Rye (Secale spp.). Bibliographia Genetica, 19, 1-86.

LEVAN, A. 1942. Studies in the meiotic mechanism of haploid Rye. Hereditas, 28, 177-211.

LEWIrsky, c. A. 1931. The morphology of chromosomes. Bull. Appl. Bot., 27, 19-174.

LIMA DE FARIA, A. 1952a. The chromomere size gradient in the chromosomes of Rye. Hereditas, 38, 246-248.

LIMA DE FARIA, A. 1952b. Chromomere analysis of the chromosome complement of Rye. Chromosoma (Berl.), 5, 1-68.

LIMA DE FARIA, A. 1959. Differential uptake of tritiated thymidine into the hetero- and euchromatin of Melanoplus and Secale. 7. Biophis. Biochem. Cyt., 6, 457-466.

MERKER, A. 1973. A Giemsa technique for rapid identification of chromosomes in Triticale. Hereditas, 75, 280-282.

NAKAO, M. 1911. Cytological studies in the nuclear division of P.M.C.'s of some cereals and their hybrids. 7. Coll. Agric. Tohoku Imp. Univ., 4, 173-190.

Nikolaieva, A. G. 1924. Notes on the cytology of Rye-Wheat hybrids. Nauchno-agron Zh., 9-10, 570-576.

pathaK, G. N. 1940. Studies in the cytology of cereals. F. Genet. 39, 437-467.

SAKamura, $r$. 1918. Kurze Mitteilung über die Chromosomeszahlen u. des Verwandschaftverhältnisse der Triticum-Arten. Bot. Mag (Tokyo), 32, 151-154.

SARMA, N. P., AND NATARAJAN, A. r. 1973. Identification of heterochromatic regions in the chromosomes of Rye. Hereditas, 74, 233-238.

stolze, к. 1925. Chromosomenzahlen der Hauptsächlichsten Getreidearten nebst algemeinen Betrachtungen Chromosomenzahl u. Chromosomengrösse im Pflanzenreich. Bibliotheca Genetica, 8, 8-71. 
SYBENGA, J., AND wOLTERS, A. H. G. 1972. The classification of the chromosomes of Rye (Secale cereale L.): a translocation tester set. Genetica, 43, 453-464.

VERMA, s. C., AND REES, H. 1974. Giemsa staining and the distribution of heterochromatin in Rye chromosomes. Heredity, 32, 118-122.

voSA, c. G., AND MARCHI, P. 1971. Quinacrine fluorescence, Giemsa staining and heterochromatin in plants. Nature, 237, 191-192.

VOSA, c. G., AND MARCHI, P. 1972 . On the Quinacrine fluorescence and Giemsa staining patterns of the chromosomes of Vicia faba. Giorn. Bot. Ital., 106, 151-159. 